\title{
Rare Perilunate Injury as a Result of Chronic Trauma in 3-Year-Old Girl
}

\author{
Ryszard Tomaszewski ${ }^{1}$ \\ ${ }^{1}$ Department of Pediatric Traumatology and Orthopedic, Silesian \\ Medical University, Katowice, Poland \\ Eur J Pediatr Surg Rep 2015;3:94-97.
}

Address for correspondence Ryszard Tomaszewski, Department of Pediatric Traumatology and Orthopedic, Silesian Medical University, Medykow16, Katowice 40-752, Poland (e-mail: tomaszewski_r@poczta.onet.pl).

\begin{abstract}
Keywords

- child perilunate dislocation

- chronic trauma

- wrist child injury

A 3-year-old girl was reported to have perilunate dislocation. The child had suffered, approximately 18 months before, a repetitive wrist injury by means of frequent pulling and bending of the hand by the father who may have physically abused the child for approximately 2 months. At the age of 3 years and 4 months, we performed the reduction of the perilunate dislocation and the plasty of the dorsal scapholunate ligament using the capsular flap prepared from the extensor retinaculum. The wrist was immobilized for 6 weeks. Two months after surgery, patient started the physiotherapy for 3 months. The patient could flex up the wrist to 40 degrees and extend up to 45 degrees (mobility of the healthy wrist was 60,50 degrees) during the examination 24 months' post-op. Only the surgical treatment of the perilunate dislocation can lead to obtain the good surgical result.
\end{abstract}

\section{New Insights and the Importance for the Pediatric Surgeon}

This article explores a radiological and surgical investigation of the perilunate wrist dislocation caused by chronic trauma in a 3-year-old child. XR, MRI and CT are essential for surgery preparation. Only surgical treatment of the perilunate wrist dislocation leads to obtain good results.

\section{Introduction}

The mother of a 3-year-old girl brought her child to our traumatology-orthopedic department because of the way the child's wrist had set due to forced bending. The child was reported to have suffered, approximately 18 months before, a repetitive wrist injury by means of frequent pulling and bending of the hand by the father who may have physically abused the child for about 2 months. The mother did not indicate a specific major trauma which could lead to a conclusion that the child may have sustained the injury when the girl was 18 months old.

On examination, there was diffuse swelling around the dorsum of the wrist, and the flexum approximately 20 degrees and the ulnar deviation approximately 20 degrees. She had normal sensation of the hand and good radial and ulnar pulses with capillary refill. On the basis of a clinical

received

November 8, 2014 accepted after revision April 6, 2015

published online

June 15, 2015 examination a computed tomography (CT) ( - Fig. 1) and a magnetic resonance imaging (MRI) ( - Fig. 2) of the wrist were consistent with the diagnosis of the perilunate dislocation, provided by the mother and the wrist MRI examination performed in our department.

The surgical treatment was applied at the age of 3 years and 4 months, the reconstruction of failured ligaments and other dislocated carpal bones were planned according to Mayfield classification. ${ }^{1}$ The medial dorsal approach reaching from the base of the distal radial bone to the base of the metacarpal was used between the second and the third compartments, the skin flaps were reflected to expose the extensor retinaculum, which was divided longitudinally and radiocarpal capsulotomy was made. After reduction of the dislocation, the plasty of the dorsal scapholunate ligament was performed using the capsular flap prepared from the extensor retinaculum. Stabilization was achieved by two (c) 2016 Georg Thieme Verlag KG
Stuttgart · New York 10.1055/s-0035-1552560 ISSN 2194-7619.
License terms

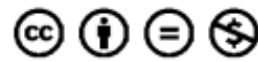




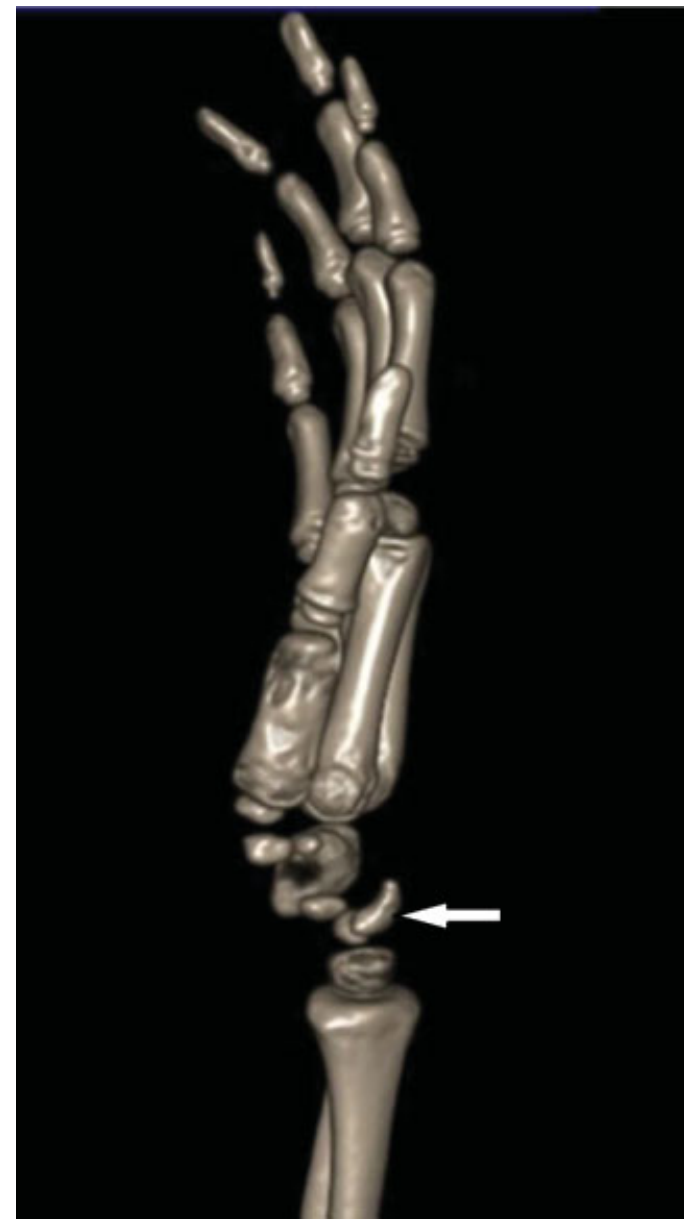

Fig. 1 Computed tomographic scan before surgery. Perilunate wrist dislocation lateral view.

Kirchner wires (K-wires) being introduced from radial and ulnar side (-Fig. 3). The wrist was immobilized using plaster cast. The K-wires were removed after 4 weeks and the wrist was immobilized in a cast for additional 2 weeks. Two months after the surgery, the patient started to undergo the physiotherapy for 3 months post-op which included gradual strengthening, movement, and proprioceptive exercises.

A medical check-up was performed after rehabilitation, 24 months after the surgery. The child did not report any pain during daily life activities or while playing sports. The hand was in a functional position with 5 degrees ulnar tilt. The patient could flex up to 40 degrees and extend up to 45 degrees (mobility of the healthy wrist was flex up to 60 degrees, extend up to 50 degrees). The control CT study at anteroposterior and lateral ( - Fig. 4) view showed a normal carpal alignment without instability and without the sign of the lunar avascular necrosis. At a lateral view, no DISI and VISI (Dorsal and Volar Intercalated Segment Instability) was found, and the scapholunaris angle was 40 degrees while the capitolunaris angle was 25 degrees (the normal is scapholunate angle in this age is 30-60 degrees and the capitolunate angle more $\geq 30$ degrees).

Because of the extremely rare type of the trauma and its being chronic in nature, the administered treatment and the

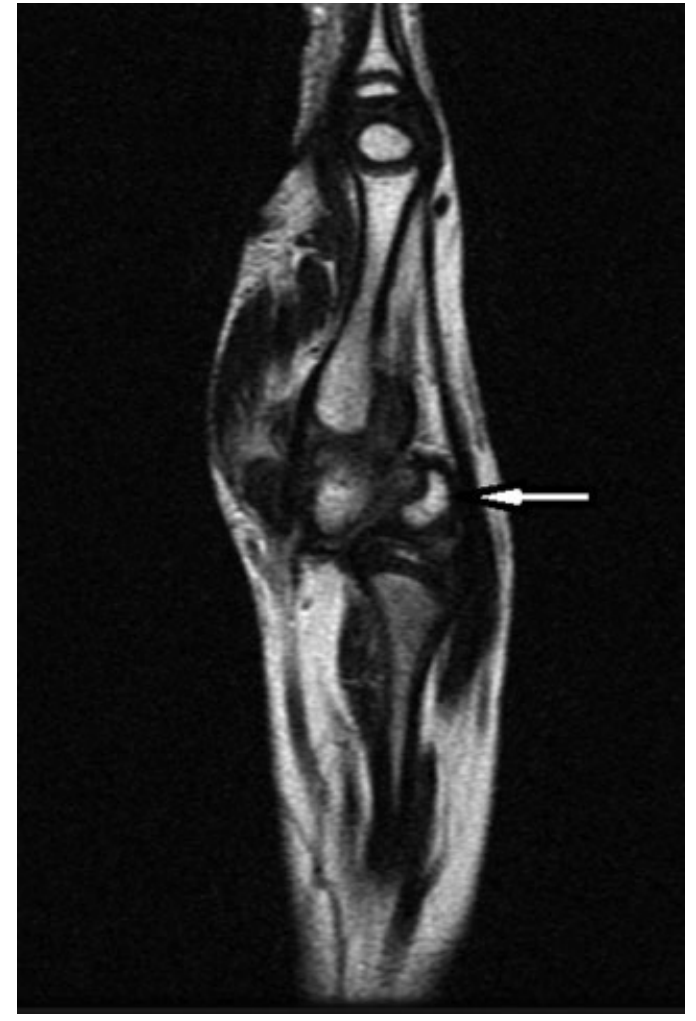

Fig. 2 Magnetic resonance imaging before surgery. Perilunate wrist dislocation lateral view.

result could be considered as good. The child and her mother were satisfied with the results of the treatment.

\section{Discussion}

The rarity of occurrence of the described fractures in children and adults ${ }^{2,3}$ does not allow for a comparison between a mechanism of sudden fractures sustained which are healed as soon as the injury is diagnosed and a fracture resulting from repeating actions leading to sprains and permanent injuries that can be qualified as old chronic trauma. The authors did not refer to any similarly described case because of the

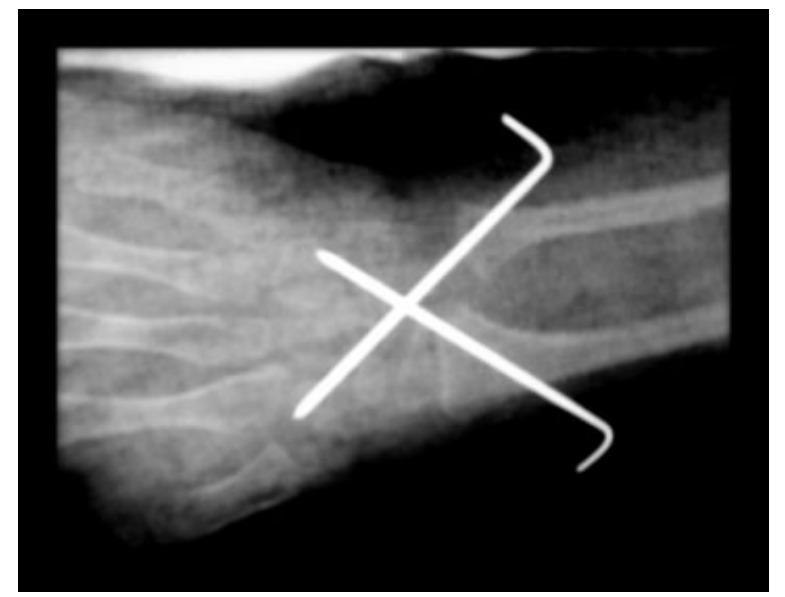

Fig. 3 X-ray with K-wires. 


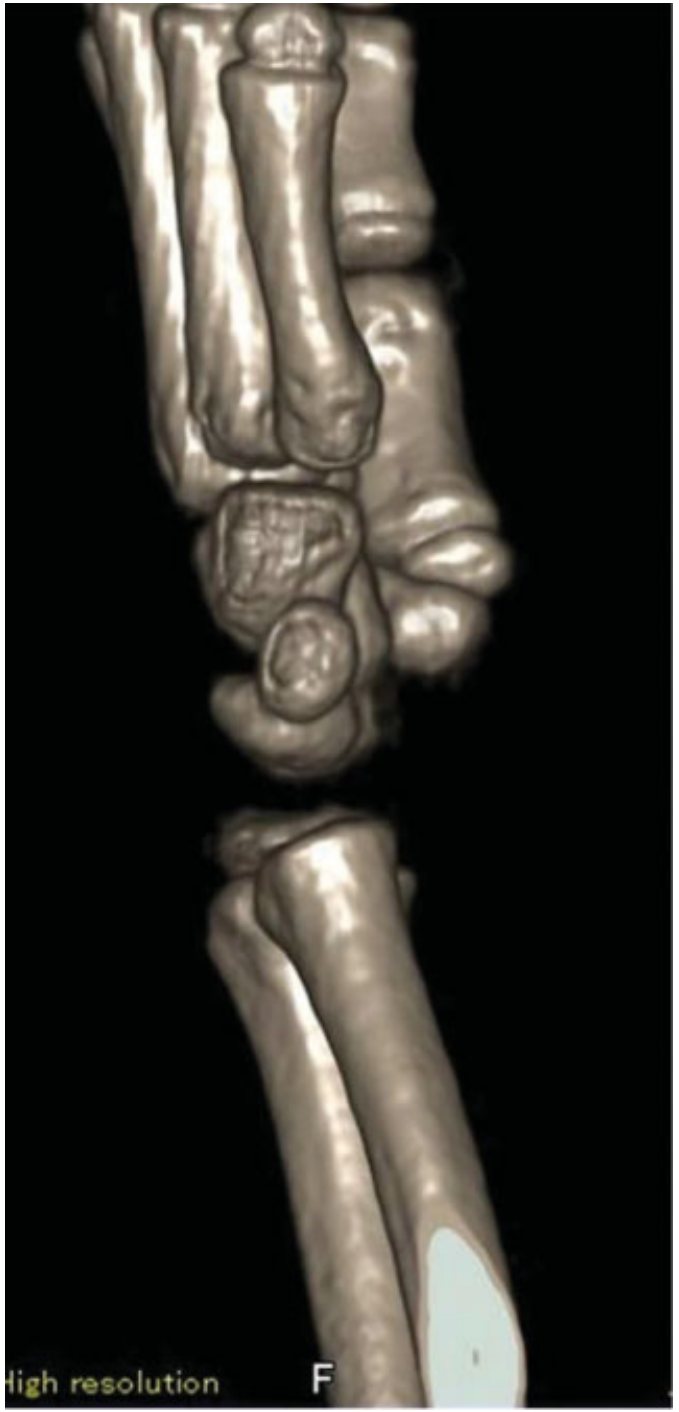

Fig. 4 Computed tomographic scan after surgery, lateral view.

apparent lack of any previous reports of this kind. However, medical literature amply describes acute perilunate dislocations ${ }^{2,4-7}$ which allows for a comparison of the type of symptoms, diagnosis, injury mechanism, treatment, effects, and further prognosis.

Acute dislocations, according to medical literature, are usually caused by injuries, often as a result of a traffic accident after a high-impact trauma, ${ }^{2}$ a fall from a great height or an injury associated with a given profession. ${ }^{4}$ In our case, there is a suspicion of a deliberate and continuous abuse of a 3-yearold girl which caused a chronic injury of a wrist. To the best of our knowledge, there is no pediatric perilunate dislocation case presentation after chronic trauma in bibliography.

The diagnosis of our case did not differ from other acute injuries described in the literature where X-ray, CT, and $\mathrm{MRI}^{2,4,5,7}$ were usually performed. Few complications have been described, although in some cases, they could be related to an insufficient reduction. ${ }^{8}$ As well as the resultant bone instability, volar lunate dislocation may also cause pressure and resultant dysfunction to the median nerve as it enters the carpal tunnel, therefore, emergency reduction of the perilunate dislocation is needed to reduce the pressure on the median nerve to try and prevent progression of nerve damage. There are also reports of the ulnar nerve being affected as a result of volar dislocation of the lunate. ${ }^{2,5}$

Secondary dislocation or instability to closed reduction and stabilization with K-wires has led many authors to advocate for primary repair through open reduction and ligament repair. ${ }^{6}$ The perilunate luxation can rarely be treated using closed reduction. ${ }^{2-8}$ But in a retrospective study, primary ligamentous repair and internal fixation with K-wires managed to maintain the anatomy of the scapholunate joint and produce better results than closed methods. ${ }^{7,8}$ The procedure in this case was performed by means of open reposition using dorsal approach and the wrist was stabilized by two K-wires.

Next, an immobilization in a plaster brachial rail for a period of 6 weeks was recommended. After 4 weeks K-wires were removed, although in the cases described in the literature such wires were removed after 6 weeks, ${ }^{4}$ and the wrist was immobilized for an additional $6^{4}$ or 8 weeks. ${ }^{5}$ Some authors, however, suggest a short period of 3 weeks of immobilization in a cast with removal of the wire after 2 weeks which allows for an early mobility of the wrist. $^{5}$ That can provide benefits to patients by speeding up the rate of functional recovery, minimizing stiffness, swelling, and pain. ${ }^{5}$ The authors prescribed rehabilitation, 4 months after the surgery the right wrist showed no compulsory settings in palmar flexion, ulnar deviation was in the range of 5 to 10 degrees, active palmar flexion was 25 degrees, and the dorsal one was 15 degrees. Physiotherapy used in our case was introduced later than in the cases described in the literature where it was used 3 months post-op. ${ }^{4}$ Because of the extremely rare type of the injury to a child as well as its late diagnosis, it is difficult to assess whether the wrist will return to its full efficiency even though there has been initially good treatment outcome. The authors claim it highly unlikely. According to the literature, ${ }^{2}$ early diagnosis and treatment are essential to maintain the range of motion and to prevent a long-term dysfunction. Multiple follow-up visits after surgery are critical as perilunate dislocations may have late complications such as instabilities, degenerative joint disease, and avascular necrosis. ${ }^{2}$ None of the reported cases in the literature ${ }^{2,4,5,7,8}$ required reoperation. In the case described by the authors of the article, no secondary surgical procedures have been made. Some authors point out that a delay in diagnosis results in the necessity of resection of the lunate. ${ }^{5}$ Despite the fact that the diagnosis in our case was delayed because of the constant and repeated damage to the bone, the bone was not damaged in such a way as to prevent a return to the full wrist fitness.

\section{Conclusion}

Treatment of perilunate dislocation is a challenging problem. Only surgical treatment can obtain good surgical result.

\section{Conflict of Interest}

None. 


\section{References}

1 Mayfield JK. Mechanism of carpal injuries. Clin Orthop Relat Res 1980;(149):45-54

2 Barbee George A, Cristóbal BC, Brandy J. Acute perilunate dislocation in a pediatric patient: Learn to recognize this uncommon but potentially devastating carpal injury. J Am Acad Physician Assist 2013;26(6):27-29

3 Panting AL, Lamb DW, Noble J, Haw CS. Dislocations of the lunate with and without fracture of the scaphoid. J Bone Joint Surg $\mathrm{Br}$ 1984;66(3):391-395

4 Ji J-H, Shafi M, Moon C-Y, Park S-E. Trans-scaphoid perilunate dislocation with fractured carpal bones in a child. Chir Main 2010; 29(1):32-35
5 Siddiqui N, Sarkar S. Isolated dorsal dislocation of the lunate. Open Orthop J 2012;6:531-534

6 Herzberg G, Comtet JJ, Linscheid RL, Amadio PC, Cooney WP, Stalder J. Perilunate dislocations and fracture-dislocations: a multicenter study. J Hand Surg Am 1993;18(5): 768-779

7 Park MJ, Steinberg DR. Volar perilunate dislocations: possible association with prior wrist injuries. Hand (NY) 2012;7(2): 217-220

8 Lee BJ, Kim SS, Lee SR, Jin JM, Yoon MG, Moon MS. Palmar scaphoid dislocation associated with dorsal perilunate dislocation: case report. J Hand Surg Am 2010;35(5): 726-731 